\title{
Caenorhabditis elegans as an alternative in vivo model to determine oral uptake, nanotoxicity, and efficacy of melatonin-loaded lipid-core nanocapsules on paraquat damage
}

\author{
This article was published in the following Dove Press journal: \\ International Journal of Nanomedicine \\ 10 August 2015 \\ Number of times this article has been viewed
}

\author{
Mariele Feiffer Charão ${ }^{1,2}$ \\ Caroline Souto ${ }^{2}$ \\ Natália Brucker ${ }^{1,2}$ \\ Anelise Barth ${ }^{1,2}$ \\ Denise $S$ Jornada ${ }^{1,3}$ \\ Daiandra Fagundez ${ }^{4}$ \\ Daiana Silva Ávila ${ }^{4}$ \\ Vera L Eifler-Lima 1,5 \\ Silvia S Guterres 1,3 \\ Adriana R Pohlmann ${ }^{1,6}$ \\ Solange Cristina Garcia ${ }^{1,2}$ \\ 'Post-Graduate Programme \\ in Pharmaceutical Sciences, \\ ${ }^{2}$ Laboratory of Toxicology (LATOX), \\ Department of Analysis, Pharmacy \\ Faculty, ${ }^{3}$ Department of Production \\ and Control of Drugs, Pharmacy \\ Faculty, Federal University of \\ Rio Grande do Sul, Porto Alegre, \\ ${ }^{4}$ Research Group in Biochemistry and \\ Toxicology in Caenorhabditis elegans \\ (GBToxCE), Federal University of \\ Pampa - UNIPAMPA, Uruguaiana, \\ ${ }^{5}$ Laboratory of Medical Synthesis \\ Organic (LaSOM), Pharmacy Faculty, \\ ${ }^{6}$ Department of Organic Chemistry, \\ Chemistry Institute, Federal \\ University of Rio Grande do Sul, \\ Porto Alegre, RS, Brazil
}

Correspondence: Solange Cristina Garcia Toxicology Laboratory, Pharmacy Faculty, Federal University of Rio Grande do Sul, Avenida Ipiranga 2752, Porto Alegre, RS 906I0-000, Brazil

Tel +55 5I 33085297

Fax +55 5I 33085437

Email solange.garcia@ufrgs.br

\begin{abstract}
Caenorhabditis elegans is an alternative in vivo model that is being successfully used to assess the pharmacological and toxic effects of drugs. The exponential growth of nanotechnology requires the use of alternative in vivo models to assess the toxic effects of theses nanomaterials. The use of polymeric nanocapsules has shown promising results for drug delivery. Moreover, these formulations have not been used in cases of intoxication, such as in treatment of paraquat (PQ) poisoning. Thus, the use of drugs with properties improved by nanotechnology is a promising approach to overcome the toxic effects of PQ. This research aimed to evaluate the absorption of rhodamine B-labeled melatonin (Mel)-loaded lipid-core nanocapsules (LNC) by C. elegans, the application of this model in nanotoxicology, and the protection of Mel-LNC against PQ damage. The formulations were prepared by self-assembly and characterized by particle sizing, zeta potential, drug content, and encapsulation efficiency. The results demonstrated that the formulations had narrow size distributions. Rhodamine B-labeled Mel-LNC were orally absorbed and distributed in the worms. The toxicity assessment of LNC showed a lethal dose $50 \%$ near the highest dose tested, indicating low toxicity of the nanocapsules. Moreover, pretreatment with Mel-LNC significantly increased the survival rate, reduced the reactive oxygen species, and maintained the development in C. elegans exposed to PQ compared to those worms that were either untreated or pretreated with free Mel. These results demonstrated for the first time the uptake and distribution of Mel-LNC by a nematode, and indicate that while LNC is not toxic, Mel-LNC prevents the effects of PQ poisoning. Thus, C. elegans may be an interesting alternative model to test the nanocapsules toxicity and efficacy.
\end{abstract}

Keywords: C. elegans, nanotoxicology, rhodamine B-labeled polymer

\section{Introduction}

Nanotechnology has increased exponentially in order to fulfill biomedical applications. ${ }^{1}$ Polymeric nanoparticles (NPs) obtained from biodegradable polymers are being studied as carriers for drug delivery. ${ }^{2-5}$ Lipid-core nanocapsules (LNC) are a special type of particle due to the hybrid characteristics between polymeric and lipid NP. ${ }^{6}$ These particles have an organogel core formed by a mixture of sorbitan monostearate and medium chain triglycerides, which increases drug encapsulation capacity by up to 40 times. $^{7}$ Recently, it has been described that the polymeric nanocapsules, prepared with poly( $\varepsilon$-caprolactone) (PCL) and polysorbate 80 (PS80), may improve the biological action ${ }^{4,8}$ of drugs, increase the drug bioavailability, ${ }^{9}$ and in case of antioxidant substances, their encapsulation has been shown to be able to improve their 
antioxidant properties. ${ }^{4,10-12}$ Moreover, PCL is being used for this purpose due to its biodegradability and biocompatibility in biological systems. ${ }^{13}$

Furthermore, in 2006, Cruz et al $^{14}$ demonstrated the antiedematogenic effect of indomethacin ethyl ester-loaded LNC after oral administration. In contrast, no effect was observed when indomethacin ethyl ester-loaded nanoemulsion was orally administered. This study was one of the first to demonstrate the importance of the polymeric wall in nanocapsules for proper oral absorption of drugs. Recently, other studies have also shown the promise of nanocarriers with LNC for the oral administration of drugs. ${ }^{6,15}$ For instance, Frozza et al ${ }^{15}$ showed a higher accumulation of resveratrol in brain tissue after oral administration of resveratrol-loaded LNC compared to the oral administration of the drug in a hydroalcoholic solution. Likewise, Pissinate et $\mathrm{al}^{6}$ observed an increase in survival of animals infected with Toxoplasma gondii after oral treatment with pyrimethamine-loaded LNC. This result was attributed to a probable increase in the central nervous system concentration of pyrimethamine promoted by the LNC, in this important site of infection by T. gondii. $^{6}$

The uptake and distribution assessment of these nanocapsules presents an interesting challenge. Hirsjärvi et al studied lipid nanocapsules and lipid nanoemulsions in different models, in vitro uptake and distribution in mice, as well as cancer treatment. The results demonstrated that sizes and surface coating had no significant difference, showing promising effects on tumor targeting. ${ }^{16}$ Recently, a rhodamine B-conjugated PCL and rhodamine B-conjugated triglyceride were developed to prepare fluorescent LNC to obtain insights regarding their uptake in macrophagic U937 human cell line. ${ }^{17,18}$ In these studies, red fluorescent cells treated with the fluorescent-labeled LNC were observed, demonstrating the applicability of both strategies of conjugation by covalent chemical bonds furnishing new fluorescent materials used to produce the dye-labeled nanocarriers for biological studies. ${ }^{17,18}$ Additionally, the cellular uptake was demonstrated in the A549 cell line, indicating the internalization of this type of $\mathrm{LNC}^{19}$

The rapid increase of nanotechnology has stimulated the use of methodologies to assess the toxicological profile of nanomaterials. ${ }^{20}$ The toxicity evaluation of nanomaterials is currently becoming detrimental, once their safety is necessary for future application. Previous studies from our research group have evaluated acute and subchronic LNC toxicity in rats after intraperitoneal and intradermal administration, whose results showed the safety of this promising drug delivery system. ${ }^{21-23}$ However, the great number of new nanomaterials requires the use of in vivo models to provide the toxicological screening, ${ }^{24}$ and following the three Rs policy (to reduce, replace, and refine the animals used for this purpose) is important, ${ }^{25}$ especially with alternative in vivo models.

The use of simpler organisms to evaluate the potential toxicity and efficacy of nanomaterials is gaining attention for animal use in experiments. ${ }^{25}$ In this line, Caenorhabditis elegans, a typical invertebrate animal model, has been successfully used in toxicological evaluations of different substances. ${ }^{26-28}$ The possibility of numerous pharmacological and toxicological tests and with the vast knowledge about the stress response at a genetic level makes the nematode C. elegans a good choice for these studies. ${ }^{29}$ Additionally, C. elegans has a short life cycle, a low cost for experimental procedures, are easy to handle, and exhibit a high degree of orthology with the human genome. ${ }^{29,30}$ Recently, C. elegans has been used to evaluate the effects of different types of nanomaterials, demonstrating its importance to nanotoxicology. ${ }^{31-35}$ To the best of our knowledge, no studies about the toxicity of polymeric NPs have been reported with this alternative in vivo model. Therefore, results obtained with this alternative model are crucial to establish new approaches in nanotoxicology and to predict their effects in complex animal models.

In addition, the development of nanoencapsulated drugs has been implicated in various pathological conditions; however this strategy has not been utilized for the treatment of acute poisonings. In this scenario, paraquat (PQ) intoxication is a serious public health problem because of its high mortality index, mainly due to the lack of an effective antidote or treatment, which is associated with its wide use even in countries where its application is forbidden. ${ }^{36-40}$ One of the main mechanism for PQ toxicity is associated with the redox cycle of PQ, where formation of superoxide anion, singlet oxygen, and other reactive oxygen species (ROS) occurs. Higher levels of these ROS lead to many deleterious effects, including lipid peroxidation in cells and tissue, protein damage, and depletion of NADPH. ${ }^{40-44}$ Additionally, the main cause of deaths by PQ poisoning is due to pulmonary fibrosis. Therefore, identifying substances that could protect from oxidative damage caused by PQ is very important. ${ }^{45}$ In this line, some studies demonstrated that melatonin could be a good antioxidant in cases of PQ-poisoning. ${ }^{38,39,43,44}$

Recently, Schaffazick et $\mathrm{al}^{12}$ demonstrated that melatoninloaded nanocapsules, prepared using melatonin, caprylic/ capric triglyceride, sorbitan monooleate, Eudragit S100, and PS80, dispersed in water were beneficial in reducing 
ascorbyl free radical-induced lipid peroxidation in the brain and liver of Wistar rats due to the improvement of melatonin antioxidant properties by its incorporation into polymeric nanocapsules. Finally, the purpose of this study was to evaluate whether melatonin-loaded LNC (Mel-LNC) rhodamine B-labeled could be orally taken up by worms and to assess the potential toxicity of LNC per se in C. elegans. Additionally, the present work aimed to verify if Mel-LNC could be a suitable candidate to reduce the toxic effects induced by PQ-poisoning.

\section{Materials and methods Reagents}

Sorbitan monostearate, melatonin, and $2^{\prime}, 7^{\prime}$-dichlorofluorescein diacetate (DCF-DA) were supplied by SigmaAldrich Co. (St Louis, MO, USA). Biodegradable polymer PCL (MW $=50,000)$ was supplied by Capa (Toledo, OH, USA). Caprylic/capric triglyceride and PS80 were obtained from Delaware (Porto Alegre, Brazil). All other chemicals and solvents were of analytical or pharmaceutical grade.

\section{Preparation of LNC formulations}

The LNC were prepared by self-assembling mechanism. ${ }^{46,47}$ Briefly, at $38^{\circ} \mathrm{C}$, the organic phase $(63 \mathrm{~mL}$ acetone, 0.025 $\mathrm{g}$ melatonin, $0.092 \mathrm{~g}$ sorbitan monostearate, $0.403 \mathrm{~mL}$ caprylic/capric triglyceride, and $0.250 \mathrm{~g}$ PCL) was injected in the aqueous phase ( $50 \mathrm{~mL}$ water and $0.1925 \mathrm{~g}$ PS80), giving a turbid aqueous solution. After 10 minutes, the organic solvent was eliminated and water partially evaporated under reduced pressure (at $40^{\circ} \mathrm{C}$ ) to reach $23-24 \mathrm{~mL}$ of white opaque liquid product. The final volume was adjusted in a volumetric flask $(25 \mathrm{~mL})$ with a theoretical final drug content of $1 \mathrm{mg} / \mathrm{mL}$.

The drug-unloaded LNC were also prepared, as described above, without adding melatonin in the formulation. As control, a melatonin aqueous solution $(1 \mathrm{mg} / \mathrm{mL})$ was also prepared using $0.77 \%(\mathrm{w} / \mathrm{v})$ of PS 80 .

To prepare melatonin fluorescent-labeled PCL LNC, 10\% of PCL used in the formulation was replaced by the rhodamine B-PCL conjugate. The conjugate was prepared by a covalent coupling of rhodamine $\mathrm{B}$ (RhoB) with the terminal hydroxyl groups of PCL, as previously described by Poletto et al. ${ }^{17}$ Briefly, under inert atmosphere (argon), rhodamine B was activated with carbodiimide (ice bath) followed by the addition of a PCL solution. The reaction underwent for 5 days at room temperature. Then, the product was purified to remove nonreacted rhodamine B by preparative column chromatography. The final product was analyzed by NMR and thin-layer chromatography for chemical identification and purity analysis.

\section{Physicochemical characterization of the LNC}

Particle size analysis and distribution

The hydrodynamic mean diameter (Z-average diameters) and polydispersity index of formulations were measured, at $25^{\circ} \mathrm{C}$ by dynamic light scattering using backscatter detection at $173^{\circ}$ (Zetasizer ZS; Malvern Instruments, Malvern, UK). The formulations $(20 \mu \mathrm{L})$ were directly diluted without treatment in $10 \mathrm{~mL}$ of prefiltered (Millipore ${ }^{\circledR}, 0.45 \mu \mathrm{m}$ ) ultrapure water.

The volume-weighted mean diameter $(\mathrm{D}[4,3])$ was determined by laser diffractometry at $25^{\circ} \mathrm{C}$ (Mastersizer 2000; Malvern Instruments) inserting each white opaque liquid product (LNC, Mel-LNC, and RhoB-labeled Mel-LNC), approximately $300 \mu \mathrm{L}$, in the dispersion accessory (wet unit) containing about $100 \mathrm{~mL}$ of distilled water. The amounts of each product were those necessary to obtain obscuration levels between 0.02 and 0.10. Background signal was discounted and measured before each analysis. In addition, the volume distribution diameter is expressed by the span value, which is determined by the mathematical relationship, calculated by Equation 1:

$$
\text { Span }=\frac{d(0.9)-d(0.1)}{d(0.5)}
$$

where $d(0.9), d(0.1)$, and $d(0.5)$ diameter are $90 \%, 10 \%$, and $50 \%$ of the cumulative distribution of diameter, respectively.

\section{$\mathrm{pH}$ measurement}

The $\mathrm{pH}$ values were determined directly in the formulation, without any dilution, using a calibrated potentiometer FiveEasy (Mettler Toledo, Brazil) at $25^{\circ} \mathrm{C}$.

\section{Zeta potential analysis}

The zeta potential was determined by electrophoretic light scattering (ZetasizerNano ZS model ZEN 3600; Malvern Instruments). The formulations were diluted 500-fold in prefiltered $0.5 \mathrm{mmol} / \mathrm{L} \mathrm{NaCl}$ (Millipore Millex-HP, $0.45 \mu \mathrm{m})$. The results were obtained from the average of three determinations.

\section{Particle number density}

The particle number density was determined by turbidimetry according to Poletto et al. ${ }^{48}$ The suspension was analyzed using 
a Cary 50 UV-Vis spectrophotometer (Varian Inc., CA, USA) in a wavelength where no photon absorption occurred (395 nm).

\section{Drug assay}

Mel-LNC suspensions were treated with acetonitrile to dissolve all components. The assay of melatonin was performed by high-performance liquid chromatography using a previously validated methodology. ${ }^{49}$ For this purpose, a PerkinElmer Series 200 chromatograph (PerkinElmer Inc., Waltham, MA, USA) was used, equipped with an ultravioletvisible detector; melatonin was quantified setting the detector at $\lambda=229 \mathrm{~nm}$. A guard-column and a column Nova-Pak C-18 (150×4.9 mm, $4 \mu \mathrm{m}$ - Waters) were employed; a flow rate of $0.7 \mathrm{~mL} / \mathrm{min}$ and an isocratic mobile phase of acetonitrile:water $(55: 45, \mathrm{v} / \mathrm{v})$ were used.

\section{Encapsulation efficiency}

Encapsulation efficiency (EE\%) was determined by pouring the formulation (Mel-LNC) into an ultrafiltration-centrifugation unit (Amicon ${ }^{\circledR}$ Ultra; EMD Millipore, Billerica, MA, USA, cutoff 10,000 Da), centrifuging (10 minutes, 15,300× $\mathrm{g}$ ), and injecting the ultrafiltrate in the high-performance liquid chromatography system without any dilution, according to the method previously described, to determine the free drug concentration $\left(C_{\mathrm{f}}\right)$. The EE\% was calculated using Equation 2, where $C_{\text {total }}$ means the total drug concentration in the formulation.

$$
\mathrm{EE} \%=\frac{C_{\text {total }}-C_{\mathrm{f}}}{C_{\text {total }}} \times 100
$$

\section{Characterization of exposure medium}

To evaluate whether the exposure medium destabilize the formulations, we preincubated both formulations with the exposure medium for 30 minutes (period of treatment), and the particle size and size distribution (span value) was measured by laser diffractometry at $25^{\circ} \mathrm{C}$ (Mastersizer 2000; Malvern Instruments), as described above. The particle size was also analyzed for pure stock suspensions. Exposure medium was prepared for the lowest and highest dose tested to verify whether there was an immediate dose-dependent difference in the dispersed particles sizes.

\section{C. elegans strain, culture and synchronization}

The nematode strain used was N2 (wild type), originally obtained from the Caenorhabditis Genetics Center (University of Minnesota, Twin Cities, MN, USA), which was maintained on nematode growth medium (NGM) plates seeded with Escherichia coli OP50 at $20^{\circ} \mathrm{C}$. Synchronization was achieved by washing off the gravid nematodes from plates to centrifuge tubes, which were lysed with a bleaching mixture $(1 \% \mathrm{NaOCl} ; 0.25 \mathrm{M} \mathrm{NaOH})$, followed by flotation on a sucrose solution $30 \%(\mathrm{~m} / \mathrm{v})$ to separate eggs from dissolved worms and bacterial debris. Eggs were washed with M9 buffer $\left(0.02 \mathrm{M} \mathrm{KH}_{2} \mathrm{PO}_{4}, 0.04 \mathrm{M} \mathrm{Na}_{2} \mathrm{HPO}_{4}, 0.08 \mathrm{M} \mathrm{NaCl}\right.$, and $0.001 \mathrm{M} \mathrm{MgSO}_{4}$ ) and allowed to hatch overnight in NGM agar plates without bacteria. ${ }^{50}$

\section{Evaluation of absorption and distribution of Mel-LNC in C. elegans}

Worms' treatment and Mel-LNC uptake

L1 synchronized worms were treated for 30 minutes with rhodamine B-labeled Mel-LNC. After the treatment period, the worms were rinsed three times with $0.9 \% \mathrm{NaCl}(\mathrm{m} / \mathrm{v})$, and 20 worms were analyzed immediately and 3 hours posttreatment. The fluorescence microscope (Olympus IX71; Olympus Corporation, Tokyo, Japan) equipped with an excitation filter of 510-550 nm connected to a digital camera (Olympus DP72; Olympus Corporation) was used to obtain the images. The assays were performed analyzing three independent experiments.

\section{Toxicity evaluation of unloaded LNC}

The toxicity of the unloaded LNC were evaluated through the toxicological endpoints in C. elegans: lethal dose $50 \%$ $\left(\mathrm{LD}_{50}\right)$, growth, and ROS production. Also, a PS80 group was analyzed to verify the influence of PS80 located on the LNC surface. Three replicates were performed for each test.

\section{$\mathrm{LD}_{50}$}

The $\mathrm{LD}_{50}$ of LNC was determined in C. elegans after acute exposure to six doses ranging from $2.37 \times 10^{12}$ to $118.5 \times 10^{12} \mathrm{LNC} / \mathrm{mL}$ (Table 1 ). Two thousand five hundred synchronized L1 worms per dose were treated at $20^{\circ} \mathrm{C}$ for 30 minutes by constant agitation in a rotator with each dose of the compounds. After that, worms were washed three times with M9 buffer. Worms were placed on OP50-seeded NGM plates $(60 \times 10 \mathrm{~mm})$ and the number of surviving worms on each plate was verified 24 hours postexposure. The dose-response curve was obtained by plotting the number of surviving worms and the $\mathrm{LD}_{50}$ value was calculated from that curve.

\section{Body area}

Body area was evaluated in adult nematodes (20 nematodes per treatment) by measuring the flat surface area of nematodes using the AxioVision software LE (version 4.8.2.0 for windows) after treatment as described above. 
Table I Concentration of PCL and LNC used in Caenorhabditis elegans during acute treatment

\begin{tabular}{llll}
\hline & $\begin{array}{l}\text { Concentration of } \\
\text { PCL* }(\mathbf{m g} / \mathbf{m L})\end{array}$ & $\begin{array}{l}\text { Concentration of } \\
\text { LNC }(\mathbf{L N C} / \mathbf{m L})\end{array}$ & $\begin{array}{l}\text { Surface area of } \\
\mathbf{L N C}\left(\mathbf{m}^{2} / \mathbf{m L}\right)\end{array}$ \\
\hline Control & - & - & - \\
Group I & 0.05 & $2.37 \times 10^{12}$ & $0.57 \times 10^{2}$ \\
Group II & 0.1 & $4.74 \times 10^{12}$ & $1.14 \times 10^{2}$ \\
Group III & 0.25 & $11.85 \times 10^{12}$ & $2.85 \times 10^{2}$ \\
Group IV & 0.5 & $23.70 \times 10^{12}$ & $5.70 \times 10^{2}$ \\
Group V & 1 & $47.40 \times 10^{12}$ & $11.40 \times 10^{2}$ \\
Group VI & 2.5 & $118.50 \times 10^{12}$ & $28.50 \times 10^{2}$ \\
\hline
\end{tabular}

Note: *PCL was the polymer used in LNC formulation.

Abbreviations: LNC, lipid-core nanocapsules; PCL, poly( $\varepsilon$-caprolactone).

\section{ROS measurement}

For ROS measurement, 1,500 worms of each group and control group were treated following the protocol described above. After the treatment, worms were resuspended in $100 \mu \mathrm{L}$ of $\mathrm{NaCl}$ solution $0.9 \%(\mathrm{~m} / \mathrm{v})$ and transferred to a $96-$ well plate. Following, $100 \mu \mathrm{L} 0.05 \mathrm{mM}$ 2,7-dichlorofluorescein diacetate (DCF-DA; Sigma-Aldrich Co.) was added, and fluorescence was measured for 90 minutes at $485 \mathrm{~nm}$ excitation and emission $530 \mathrm{~nm}$ using a microplate reader (Spectramax Me2; Molecular Devices LLC, Sunnyvale, CA, USA). In the presence of reactive species, the DCF-DA is oxidized to the fluorescent product dichlorofluorescein (DCF). The values were expressed as percentage of fluorescence intensity relative to control wells.

\section{Evaluation of Mel-LNC and free melatonin on PQ-induced toxicity}

The commercial product Gramoxone ${ }^{\circledR}$ was utilized as the source of PQ to induce the damage in order to simulate a real poisoning with this product. $\mathrm{PQ} \mathrm{LD}_{50}$ was determined after acute exposure of doses ranging from 0.05 to $2 \mathrm{mM}$. The treatment was conducted as described above (in the " $\mathrm{LD}_{50}$ " section) to each dose of PQ. The dose-response curve was obtained by plotting the number of surviving worms, and the $\mathrm{LD}_{50}$ value was calculated from that curve. For the subsequent experiments, the concentration of PQ used was the lethal dose $30 \%$, where about $30 \%$ of worms died. For Mel-LNC and free melatonin aqueous solution (Mel) exposure, the doses chosen were those which did not cause more than $5 \%$ of death, which corresponded to $10 \mu \mathrm{g} / \mathrm{mL}$. Besides, worms were also pretreated with unloaded LNC in order to evaluate whether nanocapsules per se could have some protective effect. For all dose-response curves, scores were normalized to percentage of control (treated with M9 buffer).

In order to evaluate the survival rate of the nematodes, a pretreatment with Mel-LNC $(10 \mu \mathrm{g} / \mathrm{mL}), \operatorname{Mel}(10 \mu \mathrm{g} / \mathrm{mL})$, or LNC was performed for 30 minutes at $20^{\circ} \mathrm{C}$. After three washes with M9 buffer, all worms were exposed to PQ $0.5 \mathrm{mM}$ for 30 minutes at $20^{\circ} \mathrm{C}$; additionally, a control group was exposed to M9 buffer, and a PQ-group was exposed only to PQ. Then, worms were washed three times and placed on OP50-seeded NGM plates. Scoring of survival worms was valued 24 hours after treatments.

Body area and ROS measurement were performed as described previously in the "Body area" and "ROS measurement" sections and the treatment protocols were as described in this section. Three individual experiments were performed for all experiments.

\section{Statistical analysis}

All experiments were performed at least three times. Data are expressed as mean \pm standard deviation. Dose-response lethality curves, body area, and ROS production were generated with GraphPad Prism (GraphPad Software, Inc., La Jolla, CA, USA). Statistical analysis of significance was carried out using analysis of variance for the dose-response curves and ROS production, followed by post hoc Bonferroni test in the SPSS Statistics software (version 19). Values of $P \leq 0.05$ were considered significant.

\section{Results}

\section{Preparation and characterization of LNC}

LNC and Mel-LNC presented as homogeneous with a white opaque macroscopic aspect, while RhoB-labeled Mel-LNC showed a homogeneous light pink opaque macroscopic aspect. Particle sizing analysis by dynamic light scattering showed hydrodynamic diameters (Z-average) ranging from 201 to $220 \mathrm{~nm}$, with narrow distributions and low polydispersity indexes (Table 2). The zeta potential values ranged from -5 to $-10.4 \mathrm{mV}$. The number of particles per $\mathrm{mL}$ was calculated, and the values were $4.74 \times 10^{14} \pm 1.96 \times 10^{14}$ and $6.39 \times 10^{14} \pm 0.64 \times 10^{14}$ for LNC and Mel-LNC, respectively. The LNC and Mel-LNC surface areas were $1.14 \pm 0.06$ and 
Table 2 Physicochemical characterization of LNC and Mel-LNC (batch of three samples)

\begin{tabular}{lllllll}
\hline Formulation & D $[4,3](\mathbf{n m})$ & Span & Z-average $(\mathbf{n m})$ & PDI & Zeta potential $(\mathbf{m V})$ & pH \\
\hline LNC & $243 \pm 2$ & $1.75 \pm 0.02$ & $205 \pm 4$ & $0.10 \pm 0.02$ & $-6.4 \pm 0.6$ & $5.45 \pm 0.4$ \\
Mel-LNC & $203 \pm 3$ & $1.75 \pm 0.03$ & $203 \pm 3$ & $0.11 \pm 0.01$ & $-5.2 \pm 0.2$ & $5.58 \pm 0.3$ \\
Mel-LNC-RhodB & $305 \pm 15$ & $1.44 \pm 0.11$ & $215 \pm 3$ & $0.12 \pm 0.01$ & $-9.5 \pm 0.9$ & $5.24 \pm 0.2$ \\
\hline
\end{tabular}

Note: Results expressed as mean \pm standard deviation.

Abbreviations: PDI, polydispersity index; LNC, unloaded nanocapsules; Mel-LNC, melatonin-loaded lipid-core nanocapsules; RhoB, rhodamine B.

$1.04 \pm 0.04 \mathrm{~m}^{2} / \mathrm{mL}$, respectively. There was no significant difference $(P>0.05)$ between the particle number densities and surface area for the formulations. These results demonstrated that the addition of melatonin did not influence the physicochemical characteristics of the nanocapsules. The experimental total content of melatonin was $0.96 \mathrm{mg} / \mathrm{mL}$ with an EE\% of 39\%, as expected considering its lipophilicity.

The granulometric profiles are presented in Figure 1. These results show that exclusively unimodal nanometric populations were formed.

\section{Characterization of exposure medium}

Laser diffraction analyses showed no agglomeration of LNC and Mel-LNC suspensions after addition to the exposure M9 medium (after 30 minutes). The mean diameters ( $D[4,3])$ of the LNC and Mel-LNC formulations, after addition of the M9 medium, were $198 \pm 1$ and $199 \pm 2 \mathrm{~nm}$, and span values (size distribution) were $1.57 \pm 0.009$ and $1.60 \pm 0.01$, respectively. There was no statistical difference of the $\mathrm{D}[4,3]$ values before and after contact with the M9 medium for each formulation $(P>0.05)$.

\section{Evaluation of absorption and distribution of RhoB-labeled Mel-LNC in C. elegans}

Figure 2 shows the oral absorption and distribution of RhoB-labeled Mel-LNC. It is possible to observe that worms treated with saline solution did not present red fluorescence, discarding any type of interference in the assay (Figure 2A). Figure 2B shows red fluorescence in the worm gut after 30 minutes of the treatment with rhodamine B-labeled Mel-LNC. The uptake occurs mainly via the oral route because there is remarkable fluorescence in the worm gut immediately after the treatment. Additionally, 3 hours posttreatment, there is a migration of the fluorescence from the worm gut to other tissues, indicating distribution to the tissues (Figure 2C). The images acquired from the control group (treated with M9 buffer) confirmed that the observed fluorescence is due to fluorescent-labeled LNC. Figure 2D-F show the images of the worms in contrasting phases.

\section{Toxicity evaluation of unloaded LNC}

Figure 3 shows the percentage of survival versus logarithmic dose of nanocapsules. The $\mathrm{LD}_{50}$ for $\mathrm{LNC}$ was $105.90 \times 10^{12}$ $\mathrm{LNC} / \mathrm{mL}$, and the PS80-group did not show mortality after acute exposure. All of the tested doses were compared to the control group, which did not receive the treatment.

The size of the nematodes after treatment with LNC, assessed by the measurement of surface area of the worms, showed that only the higher dose caused a decrease in body size compared to the control group (Figure $4 ; P<0.05$ ). PS80 group showed no significant difference in surface area when compared with control group (data not shown).
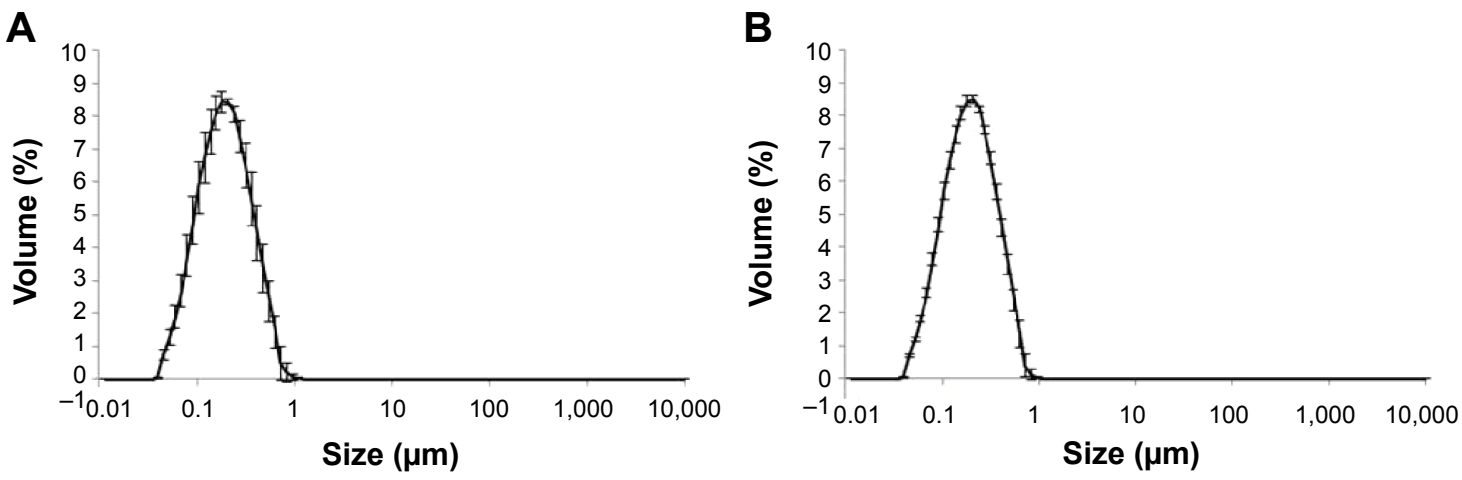

Figure I Granulometric profile (laser diffraction).

Notes: (A) Unloaded lipid-core nanocapsules. (B) Melatonin-loaded lipid-core nanocapsules. 

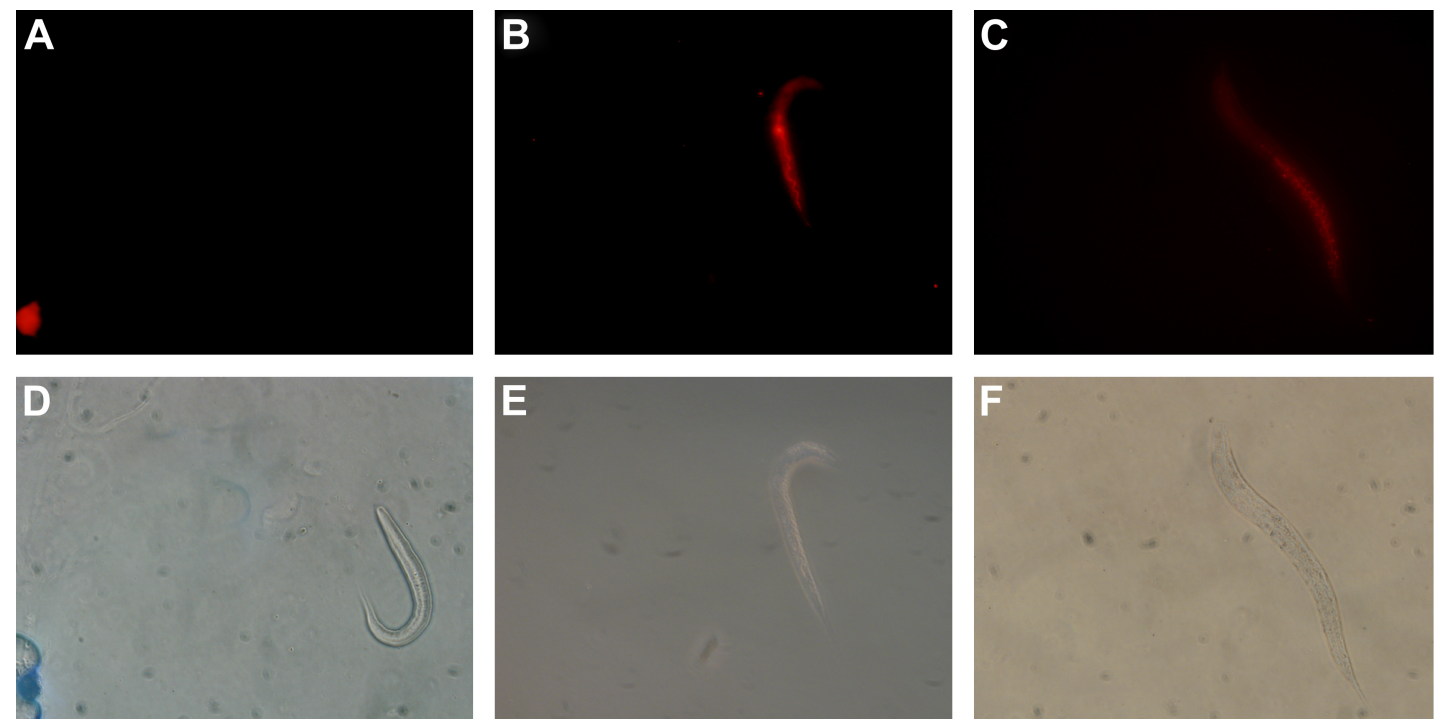

Figure 2 Microscope images (400x magnification) of worms exposed to RhoB-labeled Mel-LNC.

Notes: (A) Control worm (treated with saline solution). (B) Worm fluorescence image after 30 minutes of treatment, indicating an oral uptake. (C) Fluorescence image of worm 3 hours posttreatment, showing the distribution in the worm body. (D-F) Phase contrast microscopy images of worms; images were acquired at $400 \times$ magnification.

Abbreviations: Mel-LNC, melatonin-loaded lipid-core nanocapsules; RhoB, Rhodamine B.

ROS production following LNC exposure was performed, and the results showed that LNC caused no significant increase of ROS levels in groups I-III; however, there was a significant increase in DCF-DA oxidation in a dose-dependent manner from groups IV to VI (Figure 5, $P<0.001$ ). The PS80 group showed no increase in ROS production compared to control group (data not shown).

\section{Effects of treatment with LNC, Mel-LNC, and Mel on PQ-induced toxicity}

The acute treatment with PQ $0.5 \mathrm{mM}$ reduced the survival of the worms by approximately $30 \%$ compared to the control group, indicating the toxicity of this compound.

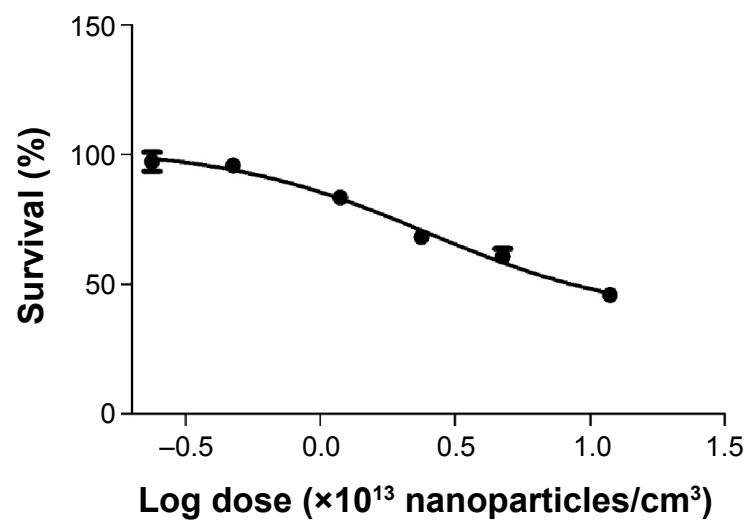

Figure 3 Log dose-response curve for lethal dose $50 \%$ determination of lipid-core nanocapsules after acute treatment.

Note: Data are expressed as mean \pm standard deviation.
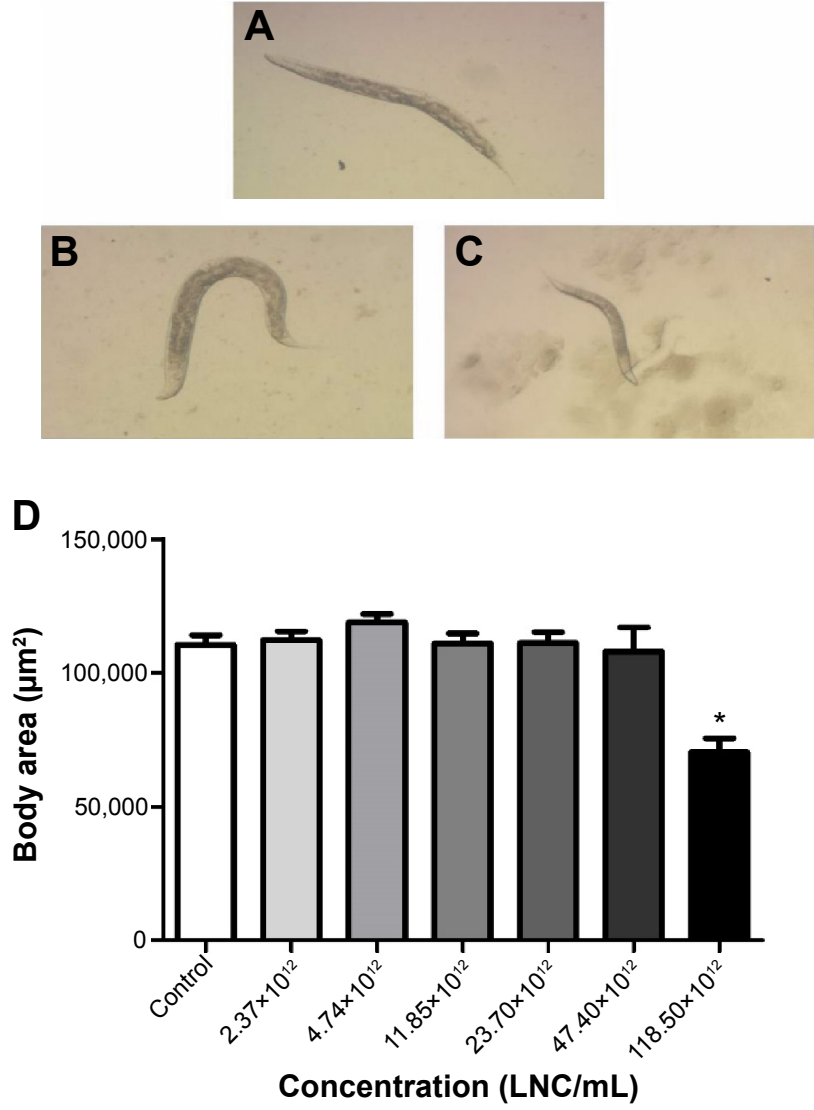

Figure 4 Body areas of Caenorhabditis elegans after acute treatment with LNC in different concentrations.

Notes: (A) Control group. (B) Group III (II.85 $\left.\times 10^{12} \mathrm{LNC} / \mathrm{mL}\right)$. (C) Group VI $\left.118.5 \times 10^{12} \mathrm{LNC} / \mathrm{mL}\right)$. (D) Graphical representation of concentration $(\mathrm{LNC} / \mathrm{mL}) \times$ body area $\left(\mu \mathrm{m}^{2}\right) . * P<0.01$ compared with control group. Abbreviation: LNC, unloaded lipid-core nanocapsules. 

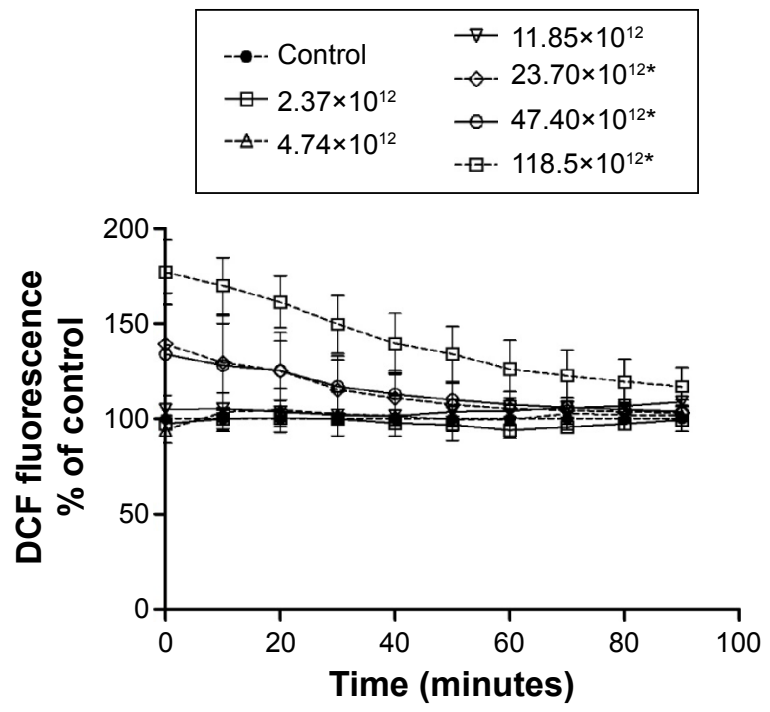

Figure 5 Reactive oxygen species production assessment after exposure with lipidcore nanocapsules in acute treatment.

Note: $* P<0.01$ compared to the control group.

Abbreviation: DCF, dichlorofluorescein.

The survival rate of the worms pretreated with Mel-LNC and then exposed to PQ was around 95\%, and significantly increased in approximately $25 \%$ compared with PQ group $(P<0.05)$; this protection was not observed with the treatment with free melatonin or LNC. These results indicate that Mel-LNC had a better protective effect than the drug solution against PQ-induced mortality in C. elegans (Figure 6).

Moreover, the normal development of $C$. elegans was affected by the PQ exposure, as it significantly reduced the body area of the worms when compared with control group $(P<0.05)$. Only the pretreatment with Mel-LNC protected the

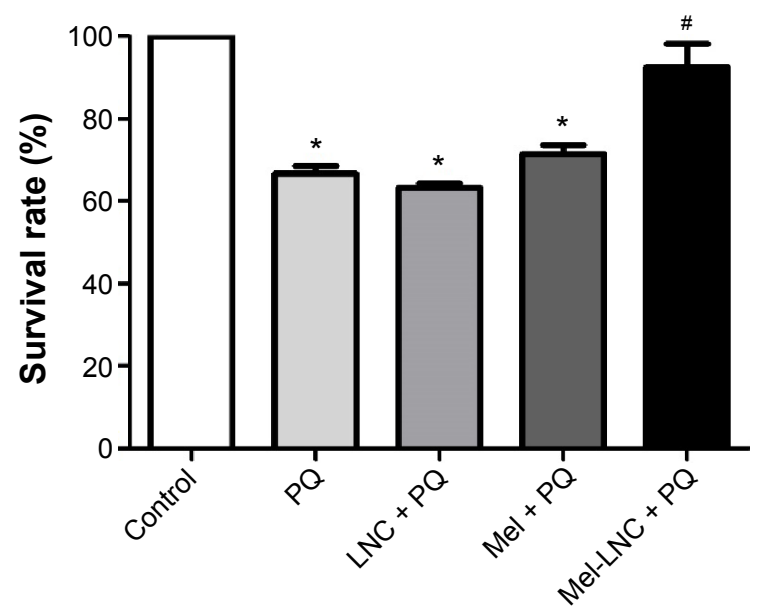

Figure 6 Survival rate of worms pretreated with Mel-LNC and Mel after PQ exposure.

Notes: Control group was exposed to M9 buffer. $* P<0.01$ compared to the control group; ${ }^{P}<0.01$ compared to the PQ group.

Abbreviations: Mel, free melatonin aqueous solution; Mel-LNC, melatonin-loaded lipid-core nanocapsules; PQ, paraquat; LNC, unloaded lipid-core nanocapsules.
PQ-exposed worms; a significant increase in the worms' body area was observed after Mel-LNC pretreatment compared to the PQ group $(P<0.05$; Figure 7$)$.

Because increased oxidative stress plays an important role in PQ toxicity, we determined the ROS levels. The analysis of ROS production was conducted through DCF-DA, which is oxidized to the DCF fluorophore in the presence of free radicals. A significant increase in DCF-DA oxidation was observed after PQ exposure (Figure $8 ; P<0.05$ ). Pretreatment with Mel-LNC significantly decreased the ROS levels induced by PQ, whereas free melatonin and LNC did not protect from the oxidative stress inflicted by the herbicide.

\section{Discussion}

In the present study, we demonstrated for the first time the internalization and distribution of drug-loaded LNC in an alternative in vivo model with $C$. elegans, and we provided toxicological evaluation of drug-unloaded LNC. Furthermore, the use of Mel-LNC was more effective than Mel in protecting C. elegans against PQ poisoning. The use of alternative methods is an important aspect because it is necessary to find animal models that are in agreement with the three Rs policy. ${ }^{25}$ C. elegans presents many advantages, among them, oral absorption is the main route of drug administration in worms. Therefore, this study demonstrated many advances, such as the use of an alternative in vivo model in which to evaluate the oral absorption as well as potential toxicity and efficacy of nanocapsules.

It is understood that the characterization of formulations is essential in efficacy and toxicity studies of a nanomaterial, and this characterization can result in reliable data and conclusions. The formulations used in this study (LNC, Mel-LNC, and RhoB-labeled Mel-LNC) presented particle mean sizes of approximately $200 \mathrm{~nm}$; these results are consistent with the diameters usually observed for this type of nanocapsule prepared using a determined amount of material by a self-assembling mechanism. ${ }^{46,47}$ The zeta potential values were negative due to the polymer and were close to zero because of PS80 coating. PS80 is a nonionic surfactant that confers stability to the formulations and prevents the aggregation of particles through a strong steric repulsive mechanism. ${ }^{46}$ A narrow particle distribution was observed for the formulations, indicated by the low polydispersity. In the melatonin-loaded nanocapsules, the total content of melatonin was $96 \%$, and the EE\% was 39\%. The change of sorbitan monooleate (liquid surfactant), previously used to prepare polymeric nanocapsules, ${ }^{51}$ by sorbitan monostearate, which was used to obtain $\mathrm{LNC},{ }^{46,47}$ did not affect the EE\%. 

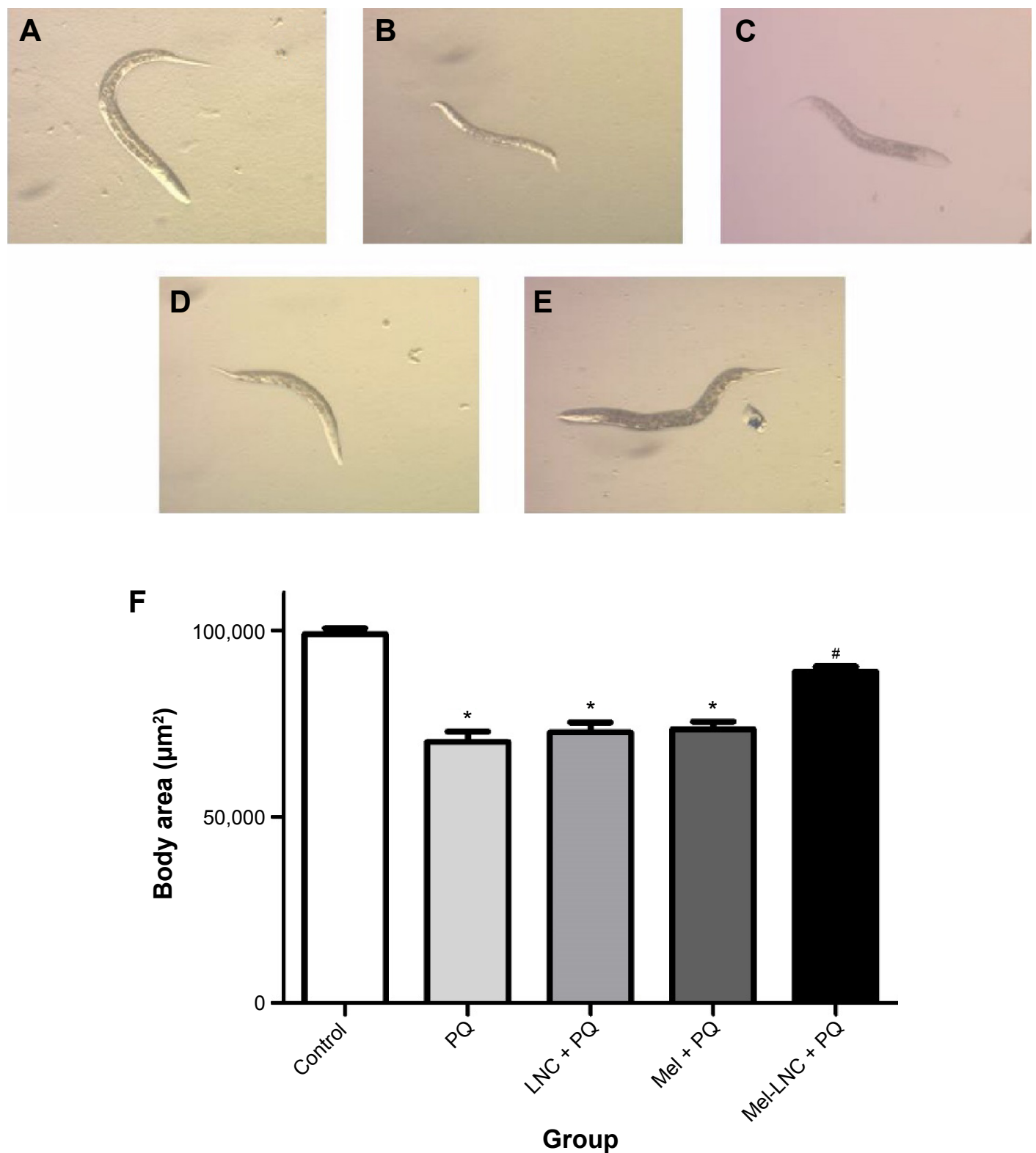

Figure 7 Mel-LNC pretreatment protected Caenorhabditis elegans from abnormal development caused by PQ-poisoning.

Notes: (A) Control group. (B) Paraquat group. (C) LNC + PQ group. (D) Mel + PQ group. (E) Mel-LNC + PQ group. (F) Graphical representation of statistic difference among the groups. ${ }^{* P}<0.01$ compared to the control group; ${ }^{*} P<0.01$ compared to the $P Q$ group.

Abbreviations: LNC, unloaded lipid-core nanocapsules; Mel, free melatonin aqueous solution; Mel-LNC, melatonin-loaded lipid-core nanocapsules; PQ, paraquat.

In addition, the medium used for worms' exposures (M9) did not interfere with the stability of the formulations, showing no alteration in the particle mean diameter $(\mathrm{D}[4,3])$ and size distribution (span) in both LNC and Mel-LNC. The results indicated no agglomeration or aggregation of the NPs in the colloidal suspensions.

The uptake of the substances is an essential prerequisite for their systemic effects. Recently, the cellular uptake and distribution of lipid nanocapsules prepared with polyethylene glycol and marked with a fluorescent dye was demonstrated, showing LNC internalization and targeting to tumor cells in vivo, and the accumulation of this formulation depends on the vascularization of the tumor. ${ }^{16}$ Our strategy to observe LNC uptake in C. elegans was to label them using a dye conjugate. We prepared rhodamine B-labeled Mel-LNC by synthetizing the rhodamine B-PCL conjugate and using it as the polymer wall in Mel-LNC, as previously proposed for drug-unloaded LNC as an approach for biological studies. ${ }^{17}$ To our knowledge, this is the first time that the uptake of LNC has been demonstrated in this in vivo system, confirming their internalization and distribution. This is an important point because approximately $40 \%$ 

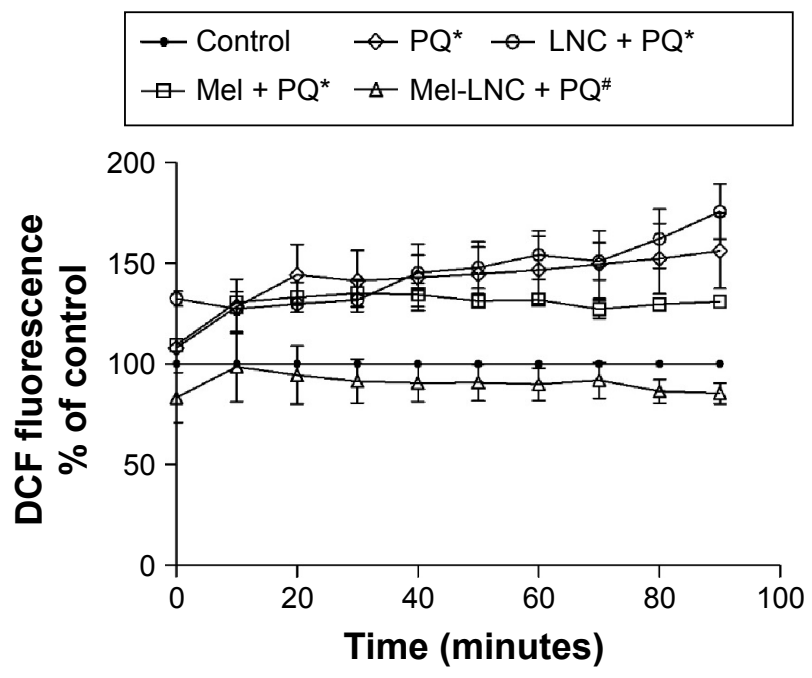

Figure 8 Pretreatment with Mel-LNC protected against PQ-induced ROS production in Caenorhabditis elegans.

Notes: ROS were measured by DCF-DA assay. $* P<0.01$ compared to control group (treated with saline solution). ${ }^{\#}<0.01$ compared to the Mel $+P Q$ group and $\mathrm{PQ}$ group.

Abbreviations: DCF, dichlorofluorescein; DCF-DA, 2',7'-dichlorofluorescein diacetate; LNC, unloaded lipid-core nanocapsules; Mel, free melatonin aqueous solution; Mel-LNC, melatonin-loaded lipid-core nanocapsules; PQ, paraquat; ROS, reactive oxygen species.

of melatonin in the formulation is incorporated into the nanocapsules. The images clearly showed the route of fluorescent nanocapsules in C. elegans; an intense fluorescence was initially observed in the gut, indicating that absorption occurs mainly via oral uptake. In addition, 3 hours posttreatment, many fluorescent dots were observed throughout the worm body, indicating the distribution of the nanocapsules to other tissues.

Due to the promising applications of nanotechnology, nanotoxicological studies are gaining prominence for the need to determine the toxicity of these nanomaterials. ${ }^{20,54}$ In vitro and in vivo studies reported that the large surface area of NPs results in a high reactivity, which could lead to cytotoxicity. ${ }^{16,20-22,54}$ The NPs' properties such as chemical composition, shape, size, surface chemistry, and large surface reactivity are mainly responsible for the possible toxicological effects caused by NPs. ${ }^{16,21,22}$ Thus, although the present work did not explore different physical and chemical characteristics of nanocapsules, this alternative model is recommended for toxicological screening because it allows a systematic evaluation of many particularities of nanomaterials, yielding rapid and accurate results.

The potential toxicity of drug-unloaded LNC was evaluated in nematodes, which were exposed to six different concentrations, and survival rate was assessed as an endpoint test that is easily observed under microscope inspection. The doses were five-times higher than the doses used in our previous studies with Wistar rats, ${ }^{21-23}$ because our purpose was the potential treatment against acute intoxication, when high doses of antidotes are necessary to reverse symptoms quickly. It was observed that increasing the dose of $\mathrm{LNC} / \mathrm{mL}$ led to an increase in mortality rate in the nematodes. Notably, it was verified that the $\mathrm{LD}_{50}$ was near the highest dose tested, indicating the low toxicity of the nanocapsules, and the concentration of $\mathrm{LNC} / \mathrm{mL}$ is higher than the concentration used in this study against PQ toxicity. These toxicity data are in agreement with our previous studies with Wistar rats, which demonstrated no toxic effects or death after acute and subchronic intraperitoneal and intradermal administration of LNC. . $^{21,22}$

Another toxicity endpoint used to verify potential toxic effects of LNC was the evaluation of the worm development, which was monitored by measuring body area. Because growth of $C$. elegans is determined by a conservative genetic regulatory pathway, this endpoint test is a good parameter to evaluate toxic effects in C. elegans. ${ }^{55}$ In the present study, we observed a significant reduction in body area between the control group and group VI $\left(118.50 \times 10^{12}\right.$ particles $\left./ \mathrm{mL}\right)$, again indicating the low toxicity of LNC. A recent study evaluated the growth of nematodes by body length after prolonged exposure to different concentrations of three metal oxide $\mathrm{NPs}_{(}\left(\mathrm{TiO}_{2} \mathrm{NPs}, \mathrm{ZnO} \mathrm{NPs}\right.$, and $\mathrm{SiO}_{2}-\mathrm{NPs}$, all with mean size of $30 \mathrm{~nm}$ ). Only exposure to $50 \mu \mathrm{g} / \mathrm{L}$ of $\mathrm{TiO}_{2} \mathrm{NPs}$ and to $\mathrm{ZnO}$ NPs significantly reduced the body lengths of nematodes. ${ }^{55}$ Another study demonstrated that the growth of nematodes was not affected by exposure to $\mathrm{CeO}_{2}$ and $\mathrm{TiO}_{2} \mathrm{NPs}^{56}{ }^{56}$ However, Cha et al showed that body length of nematodes exposed to fullerol NPs (diameter size: 4-40 nm) was significantly shorter than that of the nematodes in the control group..$^{56}$

Oxidative stress, which occurs when the generation of ROS leads to an imbalance between ROS and antioxidants, is involved in the toxicity mechanism of a large number of compounds. ${ }^{57}$ In the present study, we observed an increase in ROS production in a dose-dependent manner. In group III $\left(11.85 \times 10^{12} \mathrm{particles} / \mathrm{mL}\right)$, no significant differences were observed when compared with the control group. Otherwise, we observed a significant increase in this parameter from groups IV to VI $\left(23.70 \times 10^{12}-118.50 \times 10^{12}\right.$ particles $\left./ \mathrm{mL}\right)$, indicating a possible adverse effect caused by LNC after acute exposure. ROS-generation mechanisms are not the same for every NP, as revealed by the relationship between their toxicological effects, their type, and their physicochemical properties such as surface chemistry, particle size, shape, and composition. ${ }^{58}$

The oxidative properties of NP and ROS generation upon NP interaction with biological systems are the main factors that induce oxidative stress. ${ }^{59} \mathrm{Li}$ et al verified increased ROS 
production, determined by a DCF assay following a 10-day exposure of nematodes to $\mathrm{Al}_{2} \mathrm{O}_{3} \mathrm{NPs}(8.1-23.1 \mathrm{mg} / \mathrm{mL})$, which caused oxidative stress. ${ }^{60}$ Likewise, $\mathrm{Wu}$ et al found elevated ROS production in nematodes after prolonged exposure to $\mathrm{TiO}_{2} \mathrm{NPs}, \mathrm{ZnO} \mathrm{NPs}$, and $\mathrm{SiO}_{2} \mathrm{NPs}$ (from L1 larvae to adults), and these results demonstrated that the measurement of ROS production is a sensitive assay for assessing the toxicity of metal oxide NPs in C. elegans. ${ }^{55}$ Despite the ROS production being enhanced in groups IV-VI, LNC used in this study presented low toxicity in C. elegans once these concentrations were higher than that utilized in an experimental study with Wistar rats, which demonstrated no alterations in oxidative status. ${ }^{23}$ Once an increase in ROS production was observed in a dosedependent manner, we utilized the concentration that did not present a significant difference compared to the control group for the evaluation of protective effects of Mel-LNC.

Previous studies adopted LNC prepared with the biodegradable polymer PCL and PS80, and demonstrated that the nanoencapsulation of some substances improves their biological action. ${ }^{4,9,10,12,49,61}$ Experimental studies with these formulations have been conducted to obtain insight for cancer therapy; ${ }^{4,9,61}$ however, it is also interesting to evaluate the use of these products as a possible treatment for acute intoxications, as in PQ poisoning.

PQ is highly toxic to humans and animals, and causes severe damage to organs, such as the lungs, kidneys, and liver, ultimately resulting in death. ${ }^{39,42}$ There are no accepted guidelines for the treatment of patients intoxicated with $\mathrm{PQ}$, and the treatment normally is based on supportive care and combinations of different drugs, such as immune modulators, antioxidant therapy, hemoperfusion, and hemodialysis. ${ }^{62,63}$ Nevertheless, the mortality remains approximately $50 \%$ in hospitals that routinely practice such treatments. ${ }^{64-66} \mathrm{PQ}$ toxicity has been described in C. elegans, making this model a good choice for evaluating the protective effects of substances against PQ poisoning. ${ }^{31}$

An experimental study in which rats were intoxicated with PQ and treated with high and repeated doses of melatonin showed beneficial effects by reducing animal mortality; however, melatonin had to be used in conjunction with another substance. ${ }^{43}$ High and repeated doses of melatonin are necessary due to the short half-life and the low and variable bioavailability of melatonin.

Hence, the use of nanotechnology presents a good tool for improving the characteristics and biological properties of melatonin. ${ }^{12}$ In this context, we evaluated the effects of Mel-LNC and Mel on survival rates after PQ poisoning in C. elegans. We observed that pretreatment with Mel-LNC (single dose) significantly increased the survival rate in C. elegans exposed to PQ compared to PQ group without pretreatment or pretreated with free melatonin. These data suggest that lower doses of melatonin are necessary when the drug is encapsulated to LNC.

PQ also significantly reduced the body area of the worms, reflecting the abnormal development caused by this toxic substance. However, the pretreatment with Mel-LNC was able to protect the worms, once there was a significant increase of worms' body area when compared with the PQ group. This protection was not observed when the worms were pretreated with Mel. Melatonin exerts potent hydroxyl and peroxyl radical-scavenging activities and reduces the generation of free radicals, acting as an antioxidant. ${ }^{43,67}$ Melatonin also increases the expression of antioxidant enzymes, such as superoxide dismutase and glutathione reductase ${ }^{68}$ Thus, pretreatment with Mel-LNC was more effective in neutralizing the reactive species formed during PQ exposure, preventing the toxicity of PQ in worm development.

The toxicity mechanism of PQ has been attributed to its ability to undergo redox cycling and subsequent ROS production, resulting in several deleterious effects. ${ }^{69} \mathrm{We}$ evaluated whether Mel-LNC and Mel could reduce the ROS production caused by PQ exposure in C. elegans. The results demonstrated that $\mathrm{PQ}$ exposure at $0.5 \mathrm{mM}$ significantly increased ROS production after acute exposure compared to the control group, indicating that the ROS production is involved in the deleterious effects of PQ poisoning in C. elegans. We verified that Mel-LNC was able to significantly reduce ROS levels, but worms exposed to free melatonin did not show a difference in ROS levels compared with PQ-exposed worms. Schaffazick et al demonstrated that Mel-loaded Eudragit nanocapsules are able to reduce lipid peroxidation in mouse brains and rat livers; thus, the encapsulation of melatonin improved the antioxidant properties of this substance. ${ }^{12}$ Recently, Oliveira et al reported that Mel-LNC presented a type II distribution mechanism into suspension; approximately $40 \%$ of melatonin molecules are incorporated in the nanocapsules and $60 \%$ are dispersed in the aqueous phase. ${ }^{70}$ This type of distribution confers to the formulation a large load arrival of melatonin and a controlled release, where each nanocapsule is able to carry approximately 1,600 molecules of melatonin, explaining the more pronounced effect when compared with the melatonin aqueous solution.

\section{Conclusion}

In this study, we demonstrated the oral absorption of LNC in an in vivo alternative model based on C. elegans. Fluorescence microscopic analysis confirmed the oral 
uptake of LNC by nematodes and that it was distributed in the body of the worm. Unloaded LNC containing a biodegradable polymer (PCL) can be considered a safe formulation, corroborating previous works from our group with Wistar rats. The $\mathrm{LD}_{50}$ and normal growth of nematodes indicated low toxicity of LNC, and the ROS overproduction occurred only at high concentrations of LNC. Furthermore, Mel-LNC was able to reduce the toxic effects of PQ, increasing the survival rate and decreasing ROS generation and the adverse developmental effects. Because PQ poisoning is a serious public health problem, melatonin-loaded nanocapsules may be good candidates for studies in PQ poisoning. These results indicated that $C$. elegans may be a good alternative in vivo model for systematic toxicological screening, especially for nanotechnology studies, providing accurate and rapid results, and contributing to better exploration of the various physicochemical characteristics of nanomaterials.

\section{Acknowledgments}

This work was supported by FAPERGS, CNPq, Rede Nanotecnologia Farmacêutica CAPES, and INCT/CNPq-MCTI. A PhD research fellowship for MF Charão was provided by CAPES. SC Garcia, SS Guterres, AR Pohlmann, and V Eifler-Lima are recipients of CNPq research fellowship and Grant.

\section{Disclosure}

The authors report no conflicts of interest in this work.

\section{References}

1. Jain KK. The role of nanobiotechnology in drug discovery. Drug Discov Today. 2005;10:1435-1442.

2. Patel T, Zhou J, Piepmeier JM, Saltzman WM. Polymeric nanoparticles for drug delivery to the central nervous system. Adv Drug Deliv Rev. 2012; 64:701-705.

3. Rancan F, Blume-Peytavi U, Vogt A. Utilization of biodegradable polymeric materials as delivery agents in dermatology. Clin Cosmet Investig Dermatol. 2014;7:23-34.

4. Zanotto-Filho A, Coradini K, Braganhol E, et al. Curcumin-loaded lipidcore nanocapsules as a strategy to improve pharmacological efficacy of curcumin in glioma treatment. Eur J Pharm Biopharm. 2013;83: 156-167.

5. Mahapatro A, Singh DK. Biodegradable nanoparticles are excellent vehicle for site directed in-vivo delivery of drugs and vaccines. J Nanobiotechnology. 2011;9:55.

6. Pissinate K, dos Santos Martins-Duarte É, Schaffazick SR, et al. Pyrimethamine-loaded lipid-core nanocapsules to improve drug efficacy for the treatment of toxoplasmosis. Parasitol Res. 2014;113: 555-564.

7. Poletto FS, De Oliveira CP, Wender H, et al. How Sorbitan Monostearate Can Increase Drug-Loading Capacity of Lipid-Core Polymeric Nanocapsules. J Nanosci Nanotechnol. 2015;15:827-837.

8. Bernardi A, Frozza RL, Meneghetti A, et al. Indomethacin-loaded lipidcore nanocapsules reduce the damage triggered by A $\beta 1-42$ in Alzheimer's disease models. Int J Nanomedicine. 2012;7:4927-4942.
9. Bernardi A, Braganhol E, Jäger E, et al. Indomethacin-loaded nanocapsules treatment reduces in vivo glioblastoma growth in a rat glioma model. Cancer Lett. 2009;281:53-63.

10. Coradini K, Lima FO, Oliveira CM, et al. Co-encapsulation of resveratrol and curcumin in lipid-core nanocapsules improves their in vitro antioxidant effects. Eur J Pharm Biopharm. 2014;88:178-185.

11. Bernardi A, Frozza RL, Jäger E, et al. Selective cytotoxicity of indomethacin and indomethacin ethyl ester-loaded nanocapsules against glioma cell lines: an in vitro study. Eur J Pharmacol. 2008;586: 24-34.

12. Schaffazick SR, Siqueira IR, Badejo AS, et al. Incorporation in polymeric nanocapsules improves the antioxidant effect of melatonin against lipid peroxidation in mice brain and liver. Eur J Pharm Biopharm. 2008; 69:64-71.

13. Pohlmann AR, Fonseca FN, Paese K, et al. Poly(E-caprolactone) microcapsules and nanocapsules in drug delivery. Expert Opin Drug Deliv. 2013;10:623-638.

14. Cruz L, Schaffazick SR, Dalla Costa T. Physico-chemical characterization and in vivo evaluation of indomethacin ethyl ester-loaded nanocapsules by PCS, TEM, SAXS, interfacial alkaline hydrolysis and antiedematogenic activity. J Nanosci Nanotechnol. 2006;6:3154-3162.

15. Frozza RL, Bernardi A, Paese K, et al. Characterization of transresveratrol-loaded lipid-core nanocapsules and tissue distribution studies in rats. J Biomed Nanotechnol. 2010;6:694-703.

16. Hirsjärvi S, Dufort S, Gravier J, et al. Influence of size, surface coating and fine chemical composition on the in vitro reactivity and in vivo biodistribution of lipid nanocapsules versus lipid nanoemulsions in cancer models. Nanomedicine. 2013;9:375-387.

17. Poletto FS, Fiel LA, Lopes MV, et al. Fluorescent-Labeled Poly( $\varepsilon-$ caprolactone) Lipid-Core Nanocapsules: Synthesis, Physicochemical Properties and Macrophage Uptake. Journal of Colloid Science and Biotechnology. 2012;1:89-98.

18. Fiel LA, Paese K, Rizzi M, Guterres SS, Pohlmann AR. A strategy to estimate the intrinsic flux of a poorly water soluble substance for comparison with its release from lipid-core nanocapsules. Colloids Surf A Physicochem Eng Asp. 2014;441:716-724.

19. Charão MF, Baierle M, Gauer B, et al. Protective effects of melatoninloaded lipid-core nanocapsules on paraquat-induced cytotoxicity and genotoxicity in a pulmonary cell line. Mutat Res Genet Toxicol Environ Mutagen. 2015;784-785:1-9.

20. Doktorovova S, Souto EB, Silva AM. Nanotoxicology applied to solid lipid nanoparticles and nanostructured lipid carriers - a systematic review of in vitro data. Eur J Pharm Biopharm. 2014;87:1-18.

21. Bulcão RP, de Freitas FA, Dallegrave E, et al. In vivo toxicological evaluation of polymeric nanocapsules after intradermal administration. Eur J Pharm Biopharm. 2014;86:167-177.

22. Bulcão RP, Freitas FA, Venturini CG, et al. Acute and subchronic toxicity evaluation of poly( $\varepsilon$-caprolactone) lipid-core nanocapsules in rats. Toxicol Sci. 2013;132:162-176.

23. Bulcão RP, Bubols GB, Nascimento SN, et al. Do poly(epsiloncaprolactone) lipid-core nanocapsules induce oxidative or inflammatory damage after in vivo subchronic treatment? Toxicol Res. Epub 2015 Apr 10.

24. Fischer HC, Chan WC. Nanotoxicity: the growing need for in vivo study. Curr Opin Biotechnol. 2007;18:565-571.

25. Russel W, Burch R. The Principles of Human Experimental Technique. London: Methuen \& Co. Ltd. London; 1959.

26. Shen L, Xiao J, Ye H, Wang D. Toxicity evaluation in nematode Caenorhabditis elegans after chronic metal exposure. Environ Toxicol Pharmacol. 2009;28:125-132.

27. Roh JY, Lee J, Choi J. Assessment of stress-related gene expression in the heavy metal-exposed nematode Caenorhabditis elegans: a potential biomarker for metal-induced toxicity monitoring and environmental risk assessment. Environ Toxicol Chem. 2006;25:2946-2956.

28. Salgueiro WG, Xavier MC, Duarte LF, et al. Direct synthesis of 4-organylsulfenyl-7-chloro quinolines and their toxicological and pharmacological activities in Caenorhabditis elegans. Eur J Med Chem. 2014;75:448-459. 
29. Kaletta T, Hengartner MO. Finding function in novel targets: C. elegans as a model organism. Nat Rev Drug Discov. 2006;5:387-398.

30. Brenner S. The genetics of Caenorhabditis elegans. Genetics. 1974;77: 71-94.

31. Kim J, Takahashi M, Shimizu T, et al. Effects of a potent antioxidant, platinum nanoparticle, on the lifespan of Caenorhabditis elegans. Mech Ageing Dev. 2008;129:322-331.

32. Meyer JN, Lord CA, Yang XY, et al. Intracellular uptake and associated toxicity of silver nanoparticles in Caenorhabditis elegans. Aquat Toxicol. 2010;100:140-150.

33. Ahn JM, Eom HJ, Yang X, Meyer JN, Choi J. Comparative toxicity of silver nanoparticles on oxidative stress and DNA damage in the nematode, Caenorhabditis elegans. Chemosphere. 2014;108: 343-352.

34. Yang X, Gondikas AP, Marinakos SM, et al. Mechanism of silver nanoparticle toxicity is dependent on dissolved silver and surface coating in Caenorhabditis elegans. Environ Sci Technol. 2012;46:1119-1127.

35. Yang X, Jiang C, Hsu-Kim H, et al. Silver nanoparticle behavior, uptake, and toxicity in Caenorhabditis elegans: effects of natural organic matter. Environ Sci Technol. 2014;48:3486-3495.

36. Dinis-Oliveira RJ, Sousa C, Remião F, et al. Sodium salicylate prevents paraquat-induced apoptosis in the rat lung. Free Radic Biol Med 2007;43:48-61.

37. Kim H, Lee SW, Baek KM, Park JS, Min JH. Continuous hypoxia attenuates paraquat-induced cytotoxicity in the human A549 lung carcinoma cell line. Exp Mol Med. 2011;43:494-500.

38. Melchiorri D, Reiter RJ, Attia AM, Hara M, Burgos A, Nistico G. Potent protective effect of melatonin on in vivo paraquat-induced oxidative damage in rats. Life Sci. 1995;56:83-89.

39. Schmitt GC, Paniz C, Grotto D, et al. [General aspects and clinical laboratorial diagnostic of poisoning by paraquat]. J Bras Patol Med Lab. 2006;42:235-243. Portuguese.

40. Podder B, Kim YS, Zerin T, Song HY. Antioxidant effect of silymarin on paraquat-induced human lung adenocarcinoma A549 cell line. Food Chem Toxicol. 2012;50:3206-3214.

41. Mitsopoulos P, Suntres ZE. Cytotoxicity and gene array analysis of alveolar epithelial A549 cells exposed to paraquat. Chem Biol Interact. 2010;188:427-436.

42. Bus JS, Aust SD, Gibson JE. Lipid peroxidation: a possible mechanism for paraquat toxicity. Res Commun Chem Pathol Pharmacol. 1975;11 31-38.

43. Göcgeldi E, Uysal B, Korkmaz A, et al. Establishing the use of melatonin as an adjuvant therapeutic against paraquat-induced lung toxicity in rats. Exp Biol Med (Maywood). 2008;233:1133-1141.

44. Bonilla E, Medina-Leendertz S, Villalobos V, Molero L, Bohórquez A. Paraquat-induced oxidative stress in drosophila melanogaster: effects of melatonin, glutathione, serotonin, minocycline, lipoic acid and ascorbic acid. Neurochem Res. 2006;31:1425-1432.

45. Blanco-Ayala T, Andérica-Romero AC, Pedraza-Chaverri J. New insights into antioxidant strategies against paraquat toxicity. Free Radic Res. 2014;48:623-640.

46. Venturini CG, Jäger E, Oliveira CP, et al. Formulation of lipid core nanocapsules. Colloids Surf A Physicochem Eng Asp. 2011;375:200-208.

47. Jornada DS, Fiel LA, Bueno K, et al. Lipid-core nanocapsules: mechanism of self-assembly, control of size and loading capacity. Soft Matter. 2012;8:6646-6655.

48. Poletto FS, Jäger E, Cruz L, Pohlmann AR, Guterres SS. The effect of polymeric wall on the permeability of drug-loaded nanocapsules. Mat Sci Eng C Biomim. 2008;28:472-478.

49. Schaffazick SR, Pohlmann AR, de Cordova CA, Creczynski-Pasa TB, Guterres SS. Protective properties of melatonin-loaded nanoparticles against lipid peroxidation. Int J Pharm. 2005;289:209-213.

50. Avila DS, Benedetto A, Au C, et al. Organotellurium and organoselenium compounds attenuate Mn-induced toxicity in Caenorhabditis elegans by preventing oxidative stress. Free Radic Biol Med. 2012;52:1903-1910.
51. Schaffazick SR, Pohlmann AR, Guterres SS. Nanocapsules, nanoemulsion and nanodispersion containing melatonin: preparation, characterization and stability evaluation. Pharmazie. 2007;62:354-360.

52. KochF, Möller AM, FrenzM,PielesU,Kuehni-Boghenbor K, Mevissen M. An in vitro toxicity evaluation of gold-, PLLA- and PCL-coated silica nanoparticles in neuronal cells for nanoparticle-assisted laser-tissue soldering. Toxicol in vitro. 2014;28:990-998.

53. Zhao B, Wang XQ, Wang XY, et al. Nanotoxicity comparison of four amphiphilic polymeric micelles with similar hydrophilic or hydrophobic structure. Part Fibre Toxicol. 2013;10:47.

54. Comfort KK, Braydich-Stolle LK, Maurer EI, Hussain SM. Less is more: long-term in vitro exposure to low levels of silver nanoparticles provides new insights for nanomaterial evaluation. ACS Nano. 2014;8:3260-3271.

55. Wu Q, Nouara A, Li Y, et al. Comparison of toxicities from three metal oxide nanoparticles at environmental relevant concentrations in nematode Caenorhabditis elegans. Chemosphere. 2013;90:1123-1131.

56. Cha YJ, Lee J, Choi SS. Apoptosis-mediated in vivo toxicity of hydroxylated fullerene nanoparticles in soil nematode Caenorhabditis elegans. Chemosphere. 2012;87:49-54.

57. Sies H. Oxidative stress: oxidants and antioxidants. Exp Physiol. 1997; 82:291-295.

58. Garcia SC, Guterres SS, Bubols GB, Bulcão RP, Charão MF, Pohlmann AR In vivo toxicological evaluation, cardiotoxicity and hepatoxicity. In: Nanomedicine and Nanotechnology. 1st ed; Vol. 14. New York: Springer; 2014:299-324.

59. Manke A, Wang L, Rojanasakul Y. Mechanisms of nanoparticle-induced oxidative stress and toxicity. Biomed Res Int. 2013;2013:942916.

60. Li Y, Yu S, Wu Q, Tang M, Pu Y, Wang D. Chronic Al2O3-nanoparticle exposure causes neurotoxic effects on locomotion behaviors by inducing severe ROS production and disruption of ROS defense mechanisms in nematode Caenorhabditis elegans. J Hazard Mater. 2012; 219-220:221-230.

61. Yin $\mathrm{H}, \mathrm{Si} \mathrm{J}, \mathrm{Xu} \mathrm{H}$, et al. Resveratrol-loaded nanoparticles reduce oxidative stress induced by radiation or amyloid-beta in transgenic Caenorhabditis elegans. J Biomed Nanotech. 2014;10:1536-1544.

62. Ge W, Wang HL, Sun RP. Clinical characteristics of paraquat poisoning in 22 Chinese children. Indian J Pediatr. 2014;81:670-674.

63. Sabzghabaee AM, Eizadi-Mood N, Montazeri K, Yaraghi A, Golabi M. Fatality in paraquat poisoning. Singapore Med J. 2010;51:496-500.

64. Dawson AH, Eddleston M, Senarathna L, et al. Acute human lethal toxicity of agricultural pesticides: a prospective cohort study. PLoS Med. 2010;7:e1000357.

65. Dinis-Oliveira RJ, Duarte JA, Sánchez-Navarro A, Remião F, Bastos ML, Carvalho F. Paraquat poisonings: mechanisms of lung toxicity, clinical features, and treatment. Crit Rev Toxicol. 2008;38:13-71.

66. Wunnapuk K, Mohammed F, Gawarammana I, et al. Prediction of paraquat exposure and toxicity in clinically ill poisoned patients: a model based approach. Br J Clin Pharmacol. 2014;78:855-866.

67. Teixeira A, Morfim MP, de Cordova CA, Charão CC, de Lima VR, Creczynski-Pasa TB. Melatonin protects against pro-oxidant enzymes and reduces lipid peroxidation in distinct membranes induced by the hydroxyl and ascorbyl radicals and by peroxynitrite. $J$ Pineal Res. 2003;35:262-268.

68. Martínez-Alfaro M, Ramírez-García G, Gutiérrez-Granados S, et al. Melatonin attenuates the effects of sub-acute administration of lead on kidneys in rats without altering the lead-induced reduction in nitric oxide. J Trace Elem Med Biol. 2013;27:364-369.

69. Ranjbar A. Evidence of Oxidative Damage in Paraquat Toxicity. Zahedan Journal of Research in Medical Science. 2014;16:1-7.

70. Oliveira CP, Venturini CG, Donida B, Poletto FS, Guterres SS, Pohlmann AR. An algorithm to determine the mechanism of drug distribution in lipid-core nanocapsule formulations. Soft Matter. 2013;9: $1141-1150$ 


\section{Publish your work in this journal}

The International Journal of Nanomedicine is an international, peerreviewed journal focusing on the application of nanotechnology in diagnostics, therapeutics, and drug delivery systems throughout the biomedical field. This journal is indexed on PubMed Central,

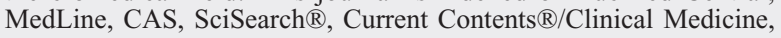

Journal Citation Reports/Science Edition, EMBase, Scopus and the Elsevier Bibliographic databases. The manuscript management system is completely online and includes a very quick and fair peer-review system, which is all easy to use. Visit http://www.dovepress.com/ testimonials.php to read real quotes from published authors.

Submit your manuscript here: http://www.dovepress.com/international-journal-of-nanomedicine-journal 